\title{
ISOLATION OF MUTANTS DEREGULATED IN PHOSPHATE CONTROL OF CANDICIDIN BIOSYNTHESIS
}

\author{
Juan F. Martín, Germán Naharro, Paloma liras and Julio R. Villanueva \\ Departamento de Microbiología, Facultades de Farmacia y Ciencias, \\ Universidad de Salamanca, Salamanca, Spain \\ (Received for publication January 16, 1979)
}

\begin{abstract}
Mutants have been isolated in which phosphate does not inhibit the biosynthesis of candicidin. At high phosphate concentrations, candicidin production by phosphate-deregulated mutants is still inhibited, but to a lesser extent than in the wild type. Some of these mutants are higher candicidin producers than the wild type, not only in phosphate-supplemented medium but also in non-supplemented production medium. The high candicidin production by these mutants is due to (1) a high specific rate of candicidin biosynthesis and (2) an extended production phase. None of the phosphate-deregulated mutants in which uptake of [ $\left.{ }^{32} \mathrm{P}\right]$ phosphate was measured was a phosphate-permeability mutant.
\end{abstract}

Inorganic phosphate exerts a depressive effect on the synthesis of many antibiotics ${ }^{3)}$. Phosphate controls the biosynthesis of antibiotics belonging to different biosynthetic groups, among them peptide antibiotics, polyene and nonpolyene macrolide antibiotics, tetracyclines and other complex antibiotics which are synthesized from different biosynthetic moieties (e.g. vancomycin) ${ }^{12}$. Generally, inorganic phosphate concentrations above $10 \mathrm{~mm}$ suppress antibiotic synthesis even though growth is stimulated by phosphate concentrations up until $300 \mathrm{~mm}$ (see review, 3). Industrial production of most antibiotics has to be carried out under phosphate-limiting conditions ${ }^{6)}$.

The biosynthesis of polyene macrolide antibiotics is extremely sensitive to phosphate in the production medium ${ }^{4}$. The molecular mechanism of the phosphate regulatory effect on the biosynthesis of candicidin has been partially elucidated. It is a complex mechanism involving repression of the biosynthesis of candicidin synthetases ${ }^{2)}$ and inhibition of the activity of the synthetases once formed ${ }^{92}$. A similar regulatory effect is exerted by exogenous nucleotides ${ }^{7},{ }^{8}$. Recently, we observed that phosphate addition to a candicidin-producing system results in a rapid increase in intracellular ATP before the inhibition of antibiotic synthesis (as measured by the incorporation of labelled precursors into candicidin) can be detected ${ }^{10)}$. This suggests that ATP may be an intracellular effector mediating control of antibiotic synthesis by phosphate. Other nucleotides, such as $3^{\prime}, 5^{\prime}$ cyclic AMP or the highly phosphorylated nucleotides ppGpp and pppGpp, do not appear to be involved in the control of onset of the synthesis of streptomycin ${ }^{1,13)}$.

It was therefore of utmost interest to isolate mutants capable of producing antibiotic in the presence of high phosphate concentrations. Such mutants should have lost the regulatory control by phosphate of candicidin biosynthesis. Moreover, mutants capable of producing antibiotic in the presence of high phosphate concentrations would have great industrial importance. Two different approaches which have been successfully applied for the isolation of such mutants are reported in this paper. A preliminary characterization of these mutants is also described. 


\section{Materials and Methods}

Microbial strains

The wild type Streptomyces griseus IMRU 3570 producer of the polyene macrolide antibiotic candicidin was used. Phosphate-deregulated (PD) S. griseus mutants were isolated according to the procedures discussed in the first paragraph of the Results section. The strains were kept lyophilised or over liquid nitrogen (gas phase) ${ }^{11)}$.

\section{Culture conditions}

Strains were cultured in SPG medium which supported high antibiotic production and which contained soya peptone $(25 \mathrm{~g} /$ liter $)$, glucose $(65 \mathrm{~g} /$ liter $)$ and zinc sulfate $(0.5 \mathrm{~mm})^{5)}$. The cultures were incubated at $32^{\circ} \mathrm{C}$ in triple-baffled $250-\mathrm{ml}$ flasks containing $50 \mathrm{ml}$ of medium in a Gallenkamp orbital shaking-incubator operating at $225 \mathrm{rev} / \mathrm{min}$. The production flasks were inoculated with $5 \mathrm{ml}$ of a 24-hour inoculum grown in YED medium containing yeast extract $(10 \mathrm{~g} / \mathrm{liter})$ and glucose $(10$ $\mathrm{g} /$ liter). The production medium was supplemented with disodium phosphate at the required concentration.

\section{Candicidin determination}

For characterization of mutants, production was measured in SPG medium solidified with $1.5 \%$ agar (Difco). Agar plugs were cut around the colonies, incubated at $28^{\circ} \mathrm{C}$ for 4 days and assayed on plates seeded with a lawn of Candida utilis prepared by pouring $15 \mathrm{ml}$ YED medium inoculated with $1 \mathrm{ml}$ of a $C$. utilis culture with an optical density of 1 . In liquid cultures candicidin was extracted with butanol and determined spectrophotometrically as indicated before ${ }^{5)}$.

\section{Uptake of labelled phosphate}

Disodium hydrogen $\left[{ }^{32} \mathrm{P}\right]$ orthophosphate, specific activity $200 \mathrm{mCi} / \mathrm{mm}$, was purchased from the Radiochemical Center (Amersham). Cells of antibiotic-producing cultures of the wild type and PD-mutants were collected at 18 hours of fermentation, washed three times with sterile saline solution and suspended in the same initial volume of fresh SPG medium. The cultures were equilibrated for 6 hours, supplemented with $10 \mathrm{~mm}$ phosphate for 10 minutes and labelled with about $5 \times 10^{5} \mathrm{cpm} / \mathrm{ml}$. Samples $(250 \mu \mathrm{l})$ were taken at intervals and added to $10 \mathrm{ml}$ of a $100 \mathrm{~mm}$ unlabelled phosphate solution directly on GF/C Whatman glass-fiber filters. After repeated washings with the unlabelled phosphate solution, the filters were dried and counted in a Packard 3320 scintillation counter.

\section{Results}

\section{Isolation of Phosphate-deregulated Mutants}

A suspension of conidia of $S$. griseus $\left(8 \times 10^{7}\right.$ conidia/ml) was mutagenized for 1.5 minutes with UV light $(254 \mathrm{~nm})$ using a Desaga ultraviolet lamp, located at a distance of $10 \mathrm{~cm}$ of the surface of the spore suspension. A $99.5 \sim 99.9 \%$ lethality was obtained in different experiments.

Two different procedures were followed after mutation. In both cases the mutated conidia were plated on complex medium (YED) to allow for phenotypic expression of the mutation. In procedure A the colonies were replicated after growth to production medium (SPG) supplemented with $10 \mathrm{~mm}$ sodium phosphate, $\mathrm{pH}$ 7.8. Agar plugs were cut out as soon as the colonies were visible and incubated for 4 days at $28^{\circ} \mathrm{C}$ in a sterile plate in water-saturated atmosphere. Production of antibiotic was assayed by the diffusion test on plates seeded with Candida utilis as test strain (Fig. 1). In the phosphate-supplemented solidified production medium most colonies produce a small inhibition zone since antibiotic production is strongly inhibited by $10 \mathrm{~mm}$ phosphate. Deregulated mutants which produce large inhibition zones in presence of $10 \mathrm{~mm}$ phosphate (Fig. 2) were found at an approximate frequency of $0.5 \sim 1 \%$. 
Fig. 1. Diagram of the two procedures used to isolated phosphate-deregulated mutants.

Mutated conidia were plated on YED medium and replicated into phosphate-containing SPGproduction medium. After incubation for 4 days the production of the antibiotic was assayed by the diffusion test in plates seeded with Candida utilis. The agar-plugs surrounded by a large inhibition zone correspond to colonies of phosphate-deregulated mutants.

Conidia (S.griseus)

U. V. Light $254 \mathrm{~nm}, 1.5 \mathrm{~m} / \mathrm{n}$.

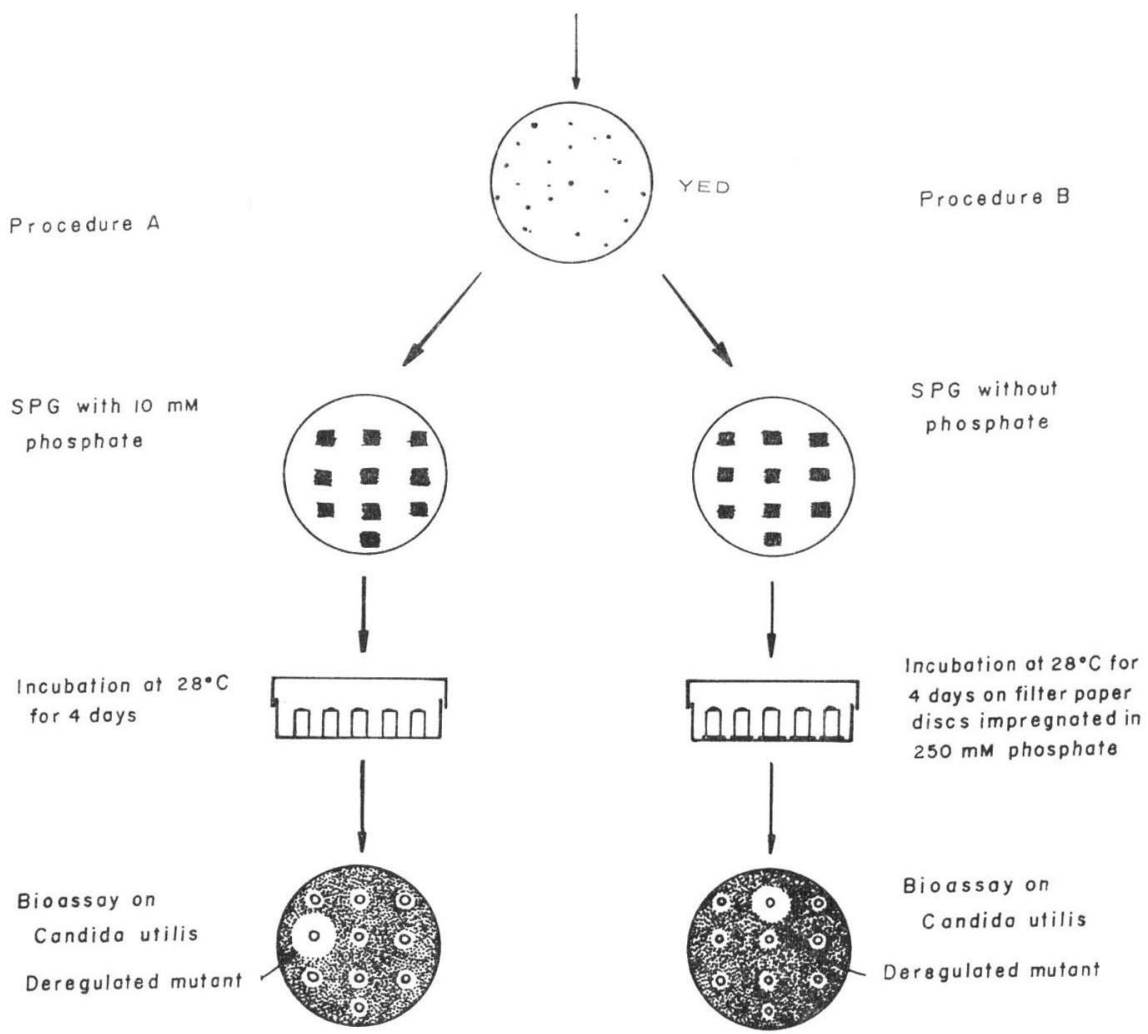

In procedure B, the colonies growing in complete medium (YED) after mutation, were replicated after growth to production medium without phosphate. Agar plugs were cut as before and incubated for 4 days in sterile plates on top of filter-paper discs saturated with $250 \mathrm{~mm}$ phosphate. Phosphate diffused from the filter-paper into the agar plug. Afterwards, the agar-plugs (without the filter paper) were transferred to the surface of assay plates seeded with a lawn of Candida utilis (Fig. 1).

Procedure B has the advantage that the colonies are transferred to high-phosphate concentration after growth has already taken place, and therefore phosphate does not affect growth but only antibiotic production. Procedure B has, however, the inconvenience that the agar plugs on the wet filter-paper discs easily become contaminated.

\section{Characterization of Phosphate-deregulated (PD) Mutants}

A large number of mutants were isolated in several experiments. Among them, the following were studied in more detail: PD-11, PD-16, PD-20, PD-21, PD-23, PD-35, PD-37, PD-44, PD-50, PD-100, PD-102, PD-103, PD-105, PD-109, PD-110, PD-120, PD-124, PD-125, PD-126, PD-128, 
Fig. 2. Isolation of phosphate-deregulated mutants. Agar plugs with colonies grown on solid SPG medium supplemented with $10 \mathrm{~mm}$ phosphate were tested for antibiotic production. The arrow points to a phosphate-deregulated mutant. The synthesis of candicidin by all other colonies is sensitive to inhibition by phosphate as it is the wild type.

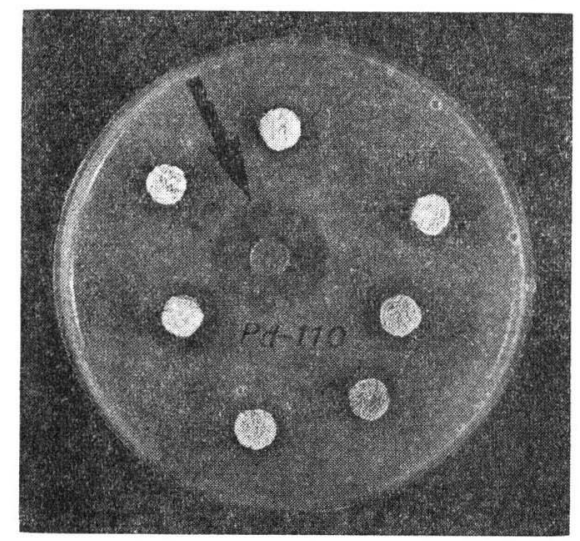

Table 1. Average diameters of the inhibition zones of the wild type and some PD-mutants in SPG medium supplemented with $10 \mathrm{~mm}$ phosphate.

\begin{tabular}{c|c}
\hline Strains & $\begin{array}{c}\text { Average diameter of } \\
\text { the inhibition zone } \\
(\mathrm{mm})\end{array}$ \\
\hline Wild type & 12 \\
PD-102 & 17 \\
PD-103 & 19 \\
PD-105 & 16 \\
PD-109 & 14 \\
PD-110 & 21 \\
PD-120 & 15 \\
PD-124 & 16 \\
PD-125 & 16 \\
PD-126 & 15 \\
PD-128 & 17 \\
PD-129 & 14 \\
PD-131 & 14 \\
PD-132 & 14 \\
\hline
\end{tabular}

Fig. 3. Characterization of phosphate-deregulated mutants.

1, wild type without phosphate supplementation. 2, wild type supplemented with $10 \mathrm{~mm}$ phosphate. 3 and 4, mutant PD-100 supplemented with $10 \mathrm{~mm}$ phosphate. 5 and 6 , mutant PD-100 without phosphate supplementation.

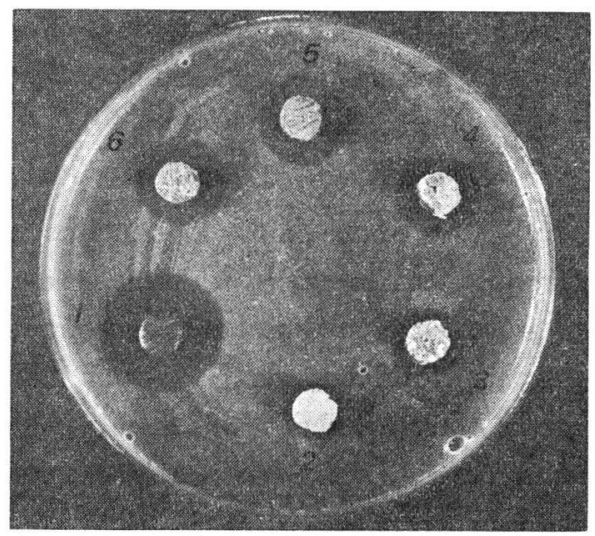

Table 2. Production of candicidin by some PDmutants in SPG medium supplemented with $25 \mathrm{~mm}$ phosphate.

\begin{tabular}{c|c|c|c}
\hline Strains & $\begin{array}{c}\text { Candicidin } \\
(\mu \mathrm{g} / \mathrm{ml})\end{array}$ & $\begin{array}{c}\text { Dry weight } \\
(\mathrm{mg} / \mathrm{ml})\end{array}$ & $\begin{array}{c}\text { Specific } \\
\text { candicidin } \\
\text { production } \\
(\mu \mathrm{g} / \mathrm{ml} \text { of } \\
\text { dry weight })\end{array}$ \\
\hline Wild type & 29.2 & 7.26 & 4.0 \\
PD-11 & 171 & 7.5 & 22.8 \\
PD-16 & 244 & 10.0 & 24.5 \\
PD-20 & 152 & 7.76 & 19.6 \\
PD-21 & 209 & - & - \\
PD-23 & 372 & - & - \\
PD-35 & 136 & 6.6 & 20.5 \\
PD-37 & 117 & 7.4 & 15.8 \\
PD-44 & 63 & 2.98 & 21.2 \\
PD-50 & 158 & 5.6 & 28.2 \\
\hline
\end{tabular}

Candicidin and dry weight were determined after 96 hours. Values are average of 3 determinations.

PD-129, PD-131 and PD-132. The diameters of the inhibition zones produced by agar plugs of several of these mutants are listed in Table 1.

All these mutants were assayed again in plates of production medium (SPG) supplemented and unsupplemented with $10 \mathrm{~mm}$ phosphate (Fig. 3). Those which overproduced in the presence of $10 \mathrm{~mm}$ phosphate were assayed in liquid cultures with and without supplementation with phosphate. Table 2 shows the growth and the production of the wild type and several of these mutants in SPG supplemented with $25 \mathrm{~mm}$ phosphate. 
These results indicate that the PD-mutants are high producers as compared to the wild type when grown in SPG medium supplemented with $25 \mathrm{~mm}$ phosphate. Mutant PD-16 grows better than the wild type whereas mutant PD-44 shows very little growth, although the specific candicidin production is high.

\section{Effect of Different Concentrations of}

Phosphate on Candicidin

Production by the Wild

Type and the PD-Mutants

As shown above, most PD-mutants were overproducers as compared with the wild type when grown in high-phosphate concentrations. It was, therefore, important to study the response of these mutants to different phosphate concentrations. Table 3 summarizes the results of candicidin production by the wild-type and some PD-mutants without supplementation with phosphate and supplemented with $5 \mathrm{~mm}$ or 10 mu phosphate.

Table 3. Effect of different concentrations of phosphate on candicidin production by some PDmutants.

\begin{tabular}{l|r|r|r}
\hline \multirow{2}{*}{ Strains } & \multicolumn{3}{|c}{$\begin{array}{c}\text { Candicidin production }(\mu \mathrm{g} / \mathrm{ml}) \text { at } \\
\text { different phosphate concentration }\end{array}$} \\
\cline { 2 - 4 } & $0 \mathrm{mM}$ & $5 \mathrm{~mm}$ & $10 \mathrm{~mm}$ \\
\hline Wild type & 1,038 & 196 & 60 \\
PD-11 & 1,770 & 511 & 193 \\
PD-26 & 885 & 540 & 153 \\
PD-50 & 1,680 & 525 & 219 \\
\hline Wild type & 948 & 290 & 65 \\
PD-21 & 904 & 587 & - \\
PD-23 & 1,168 & 440 & 226 \\
\hline Wild type & 975 & 225 & - \\
PD-110 & 1,296 & 561 & - \\
\hline
\end{tabular}

Candicidin values are average of 3 flasks at 104 hours.

These results indicate that synthesis of candicidin by PD-mutants is less sensitive to phosphate than the synthesis of candicidin by the wild type.

Particularly interesting is the observation that some PD-mutants are overproducers even without supplementation with phosphate.

\section{Kinetics of Candicidin Production by PD-Mutants under Normal Fermentation Conditions}

To know whether candicidin production by PD-mutants follows similar dynamics to that of the wild type ${ }^{\text {s) }}$, we compared the production of the wild type and the best PD-mutants in SPG medium without supplementation with phosphate. Results are indicated in Fig. 4.

Mutants PD-11, PD-50, PD-21 and PD-23 were higher producers than the wild type after 105 hours of fermentation. The more relevant characteristic is their capability to maintain a high rate of antibiotic synthesis in the late phase of fermentation when production by the wild type is slowing down.

\section{Uptake of Labelled Phosphate by PD-Mutants}

Since PD-mutants might simply be mutants altered in the cell permeability to phosphate, the uptake of $\left[{ }^{32} \mathrm{P}\right]$ phosphate by mutants PD-110, PD-21 and PD-23 was compared with that of the wild type.

The results are indicated in Fig. 5. PD-110 has a similar uptake to the wild type that was consistent in different experiments. PD-21 shows higher phosphate uptake than that of the wild type whereas PD-23 shows a slightly lower uptake that was also consistent.

The differences in uptake are not sufficient however, to explain the deregulation of candicidin synthesis. None of the PD-mutants are considered to be permeability mutants. 
Fig. 4. Kinetics of candicidin production by phosphate-deregulated mutants.

Culture conditions and candicidin determination are described in the text.

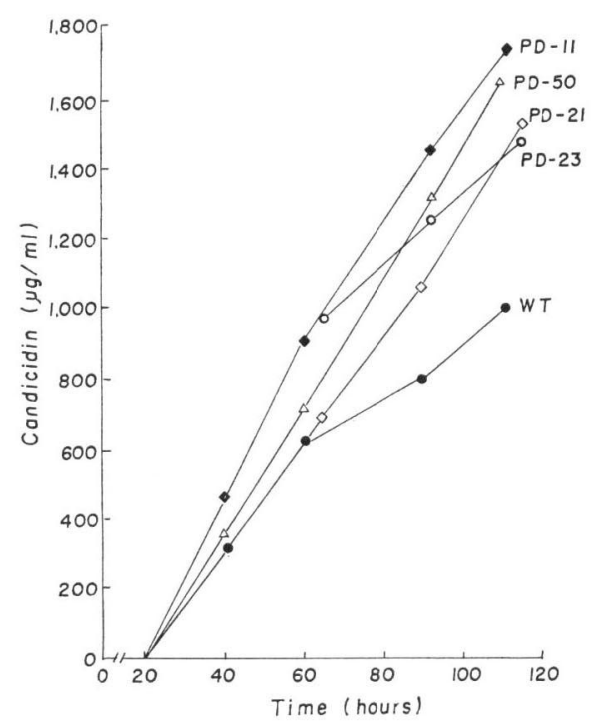

Fig. 5. Uptake of labelled phosphate by phosphatederegulated mutants. The uptake of disodium hydrogen $\left[{ }^{32} \mathrm{P}\right]$ orthophosphate was determined in cultures supplemented with $10 \mathrm{~mm}$ unlabelled phosphate for 10 minutes before addition of the radioactivity, as indicated in Methods.

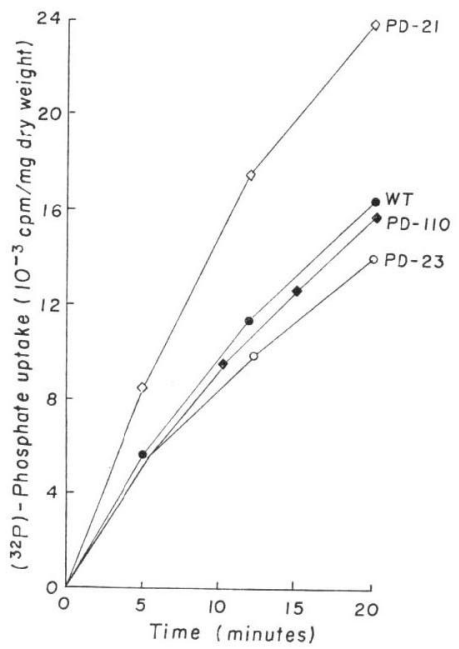

\section{Discussion}

A large number of mutants able to produce candicidin in the presence of $10 \mathrm{~mm}$ phosphate has been obtained. However, several of these mutants turned out to be rather unstable and sensitivity of candicidin synthesis to phosphate returned to normal levels after several transfers. Others seem to be genetically stable even in the absence of selective pressure. Some mutants show an altered pattern of growth, forming colonies larger than the wild type, but most PD-mutants show the same morphology and growth characteristics as the wild type.

All PD-mutants were higher producers than the wild type in liquid cultures supplemented with $25 \mathrm{~mm}$ phosphate. A very interesting result is the fact that some PD-mutants were overproducers in SPG fermentation medium without phosphate supplementation. This result indicates that normal complex fermentation media may contain levels of phosphate repressive for antibiotic synthesis. Therefore, PD-mutants which are less sensitive to phosphate regulation are derepressed for antibiotic production at phosphate levels in which the wild type is already repressed.

The kinetics of antibiotic production by PD-mutants shows that antibiotic production is delayed until after the growth phase. PD-mutants keep a high rate of antibiotic synthesis in the late phase of the fermentation unlike the wild type where synthesis of antibiotic levels off, apparently due to an inactivation of candicidin synthetases. This result indicates that the candicidin synthetases of PDmutants are more stable. It is also possible that the nutritional pattern of the fermentation favors continued stability of candicidin synthetases in PD-mutants.

None of the PD-mutants which were assayed for $\left[{ }^{32} \mathrm{P}\right]$ phosphate uptake is a permeability mutant. In fact, mutant PD-21 shows an increased phosphate uptake. Faster utilization of phosphate in this mutant may cause earlier depletion of phosphate from the culture.

Since phosphate uptake is normal in these mutants, the decreased sensitivity of candicidin synthesis to phosphate regulation may be explained on the basis of a deregulation of the enzymes involved in candicidin synthesis. Studies are now under way to characterize these mutations at the molecular level.

Phosphate-deregulated mutants may have great industrial relevance providing that adequate stable mutants are obtained. Since antibiotic production by PD-mutants is partially insensitive to 
phosphate, candicidin fermentations may be carried out in complex media with a higher phosphate content or in media supplmented with phosphate. In both cases a higher biomass would be formed. Since total production is the result of both the specific production and the biomass accumulated, cultures with a higher cell density than the wild type will give higher yields providing that specific production is maintained.

\section{References}

1) AN, G. \& L. C. VINING: Intracellular levels of guanosine 5'-diphosphate 3'-diphosphate (ppGpp) and guanosine 5'-triphosphate 3'-diphosphate (ppGpp) in cultures of Streptomyces griseus producing streptomycin. Canad. J. Microbiol. 24: 502 511, 1978

2) Liras, P.; J. R. Villanueva \& J.F. Martin: Sequential expression of macromolecule biosynthesis and candicidin formation in Streptomyces griseus. J. Gen. Microbiol. 102: 269 277, 1977

3) Martin, J. F.: Control of antibiotic synthesis by phosphate. In "Advances in Biochemical Engineering". Vol. 6. Ed. by T. K. Ghose, A. Fiechter \& N. Blakebrough. pp. 105 127, Springer-Verlag, Berlin, 1977

4) Martin, J. F.: Biosynthesis of polyene macrolide antibiotics. Ann. Rev. Microbiol. 31: 13 38, 1977

5) Martin, J. F. \& L. E. MCDaniel: Kinetics of biosynthesis of polyene macrolide antibiotics in batch cultures: Cell maturation time. Biotechnol. Bioeng. 17: 925 938, 1975

6) Martin, J. F. \& L. E. MCDaniel: Biosynthesis of candicidin by phosphate-limited resting cells of Streptomyces griseus. Eur. J. Appl. Microbiol. 3: 135 144, 1976

7) Martin, J.F. \& A. L. Demain: Effect of exogenous nucleotides on the candicidin fermentation. Canad. J. Microbiol. 23: 1334 1339, 1977

8) Martin, J. F. \& A. L. Demain: Cleavage of adenosine 5'-monophosphate during uptake by Streptomyces griseus. J. Bacteriol. 132: 590 595, 1977

9) Martin, J. F.; P. Liras \& A.L. Demain: Inhibition by phosphate of the activity of candicidin synthetases. FEMS Microbiol. Lett. 2: 173 176 1977

10) Martin, J. F.; P. Liras \& A. L. Demain: ATP and adenylate energy charge during phosphate-mediated control of antibiotic synthesis. Biochem. Biophys. Res. Commun. 83: 822 828, 1978

11) MCDaviel, L. E. \& E. G. BaIley: Liquid nitrogen preservation of standard inoculum: gas-phase storage. Appl. Microbiol. 16: 912 916, 1978

12) Mertz, F. P. \& L. E. Doolin: The effect of inorganic phosphate on the biosynthesis of vancomycin. Canad. J. Microbiol. 19: 263 270, 1973

13) RaGAN, C. M. \& L. C. VINING: Intracellular cyclic adenosine 3',5'-monophosphate levels and streptomycin production in cultures of Streptomyces griseus. Canad. J. Microbiol. 24: 1012 1015, 1978 